\title{
A global call for action to combat antimicrobial resistance: Can we get it right this time?
}

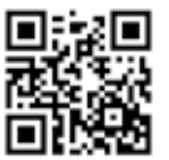

On 17 May 2014, the World Health Assembly adopted World Health Organization (WHO) resolution WHA67.25 'Combating antimicrobial resistance including antibiotic resistance. ${ }^{[1]}$ Among other directives, the nine-point call to action urges member states 'to develop or strengthen national plans, strategies and international collaboration for the containment of antimicrobial resistance'. Such calls have been heard before. ${ }^{[2-3]}$ So what is different this time, how do we break the mould to ensure that significant international change occurs, and how is South Africa (SA) positioning itself to respond to the call?

Commonly, a crisis only becomes a crisis when high-income countries become threatened and a tipping point is reached where a commodity becomes scarce. Bugs travel, and with them come antimicrobial resistance (AMR) genes. The global dissemination of New Delhi metallo-beta-lactamase-1 (NDM-1)-containing Gram-negative bacteria from India ${ }^{[4]}$ and Klebsiella pneumoniae carbapenemase (KPC) from the USA, ${ }^{[5]}$ testifies to the fact that we are facing an international problem, capable of affecting all countries irrespective of how good their antimicrobial policies to prevent resistance are. As such, an international, collaborative approach is needed where all nations are involved. To cement this, a sea-change in perception is required. The WHO resolution recognises the efficacy of an antimicrobial as a global public good, i.e. one that is both non-excludable (universal access) and non-rival in consumption (will not deplete the efficacy of the drug). The non-rival nature of antimicrobial efficacy can only be preserved if we stop perceiving antimicrobials as our own private goods, and attend to the main drivers of resistance. This is not only relevant to antimicrobials for human use, but applies equally to the far greater volume used for animal health, agriculture and aquaculture.

While antibacterial resistance is a Darwinian response by bacteria to selective pressure exerted by an antibacterial, its main driver is inappropriate overuse. This practice spans resource settings, rural and urban communities, public and private healthcare, and all types of prescribers. Empiric antibacterial prescribing for undifferentiated fever commonly lies at the heart of inappropriate use, whether it is the child in a rural low- or middle-income country attending a local clinic with what is actually malaria, adults throughout the world requesting antibacterials for themselves or their children for viral upper respiratory tract infection, or the elderly patient who is admitted to hospital with fever and confusion labelled sepsis, unknown cause'. Improved diagnostics, preferably at point of care and ideally those that identify not only the microbe but its resistance profile too, in addition to development of laboratory infrastructure and services to strengthen surveillance, would enable appropriate prescribing. Education, evidence-based guidelines and task shifting to allow safe, appropriate non-physician-based prescribing would improve access to appropriately prescribed antimicrobials to all in need, and removal of perverse incentives to over-prescribe, such as remuneration linked to prescription, would disincentivise inappropriate prescribing.

The lack of new drugs to treat bacteria containing NDM-1, KPC and other multidrug-resistant mutations is having significant effects on patient morbidity and mortality. ${ }^{[6-7]}$ For many such infections we now rely on colistin, a drug developed in the 1960s with significant renal toxicity, as the last line of defence. The increasing reports of colistin resistance, including in our own country, herald our entry into the post-antibiotic era. ${ }^{[8,9]}$ With no new antibacterials expected for multidrug-resistant Gram-negative bacteria in the next $10-15$ years based on current models, we need to preserve what we have, and reinvigorate the antibacterial drug pipeline. To enable this, new models of antimicrobial research and development (R\&D), and pricing that de-links profit from volume of sales, are required, and a combination of push-and-pull mechanisms, private-public partnerships and other innovative models that can serve as incentives for R\&D should be explored.

AMR in the SA context needs to take into account a range of infections. Extensively and totally drug-resistant tuberculosis (TB) is spreading in the country; ${ }^{[10]}$ approximately half of all non-albicans candida infections are resistant to fluconazole; ${ }^{[11]}$ current rates of transmitted resistance to first-line antiretrovirals in HIV are $~ 5 \%$, yet as antiretroviral roll-out continues, resistance rates are set to rise to $10-17 \% ;{ }^{[12]}$ and hopes for the elimination of falciparum malaria are threatened by the risk of spread of artemesinin resistance from SouthEast Asia ${ }^{[13]}$ A number of strategies are already in place for HIV, TB and malaria through established national working groups. In terms of antibacterial resistance, the national response has gathered pace since the publication of a situational analysis in 2011 by the Global Antibiotic Resistance Partnership-South Africa (GARP-SA) ${ }^{[14]}$ The South African Antibiotic Stewardship Programme (SAASP) ${ }^{[15]}$ has led the introduction of stewardship to promote appropriate antimicrobial prescribing across public and private health sectors, with positive results. ${ }^{[16]}$ AMR working groups with co-ordinated activities have begun work in some provinces, and following meetings between the SAASP and the National Department of Health, work began on a national AMR strategy with widespread stakeholder involvement. This work has accelerated since the WHO resolution was proposed in January 2014. The strategy rests on strengthening four pillars: governance, surveillance and reporting, antimicrobial stewardship (AMS), and infection prevention and control.

Recognising that AMR involves human and animal health, and requires co-ordinated collaboration with other key areas, an intersectoral Ministerial Advisory Committee is to be formed to advise the Minster of Health. The committee will include representation from the departments of Health, Agriculture, Forestry and Fisheries, Science and Technology, Trade and Industry, and Education, in addition to public and private health providers, academia, infection societies and other relevant stakeholders. National core standards for AMS and infection prevention control (IPC) are under consideration, which will direct governance at institutional and district levels. Strengthening of surveillance and reporting go beyond patterns of AMR to include antimicrobial use, drug quality and medication errors, all of which are important factors to optimise prescribing. A central, national body is needed to co-ordinate and warehouse all laboratory surveillance data, and both statutory and sentinel reporting notification systems are required to inform appropriate prescribing.

To strengthen AMS, we need to build expertise by integrating it into curricula for all healthcare professionals in training and making 
it part of continuing professional development programmes. We need to institutionalise AMS so that it is incorporated into job descriptions and performance appraisals, and develop 'champions' in facilities and districts. At the coalface, the strategy is to integrate information technology systems so that pharmacy, microbiology and clinical information can be collated and drive appropriate prescribing. Adopting national core standards that require each institution and district to have an AMS committee and AMS team(s) will promote appropriate prescribing, as will a set of national guidelines, in line with the Essential Drugs List and structured treatment guidelines. For animal health, we need to phase out the use of antimicrobials where evidence exists that they select out for cross-resistance in humans. In addition, a co-ordinated programme of research and audit is required towards phasing out of antimicrobials used for animal growth promotion.

The strategy to strengthen IPC mirrors much of that for AMS in terms of education, clear governance structures, and integration with AMS team activities. Supporting the Expanded Program on Immunization to ensure that our children, high-risk adolescents and adults are vaccinated against bacterial and viral infections can reduce the need for antimicrobials in the first place. Creating the infrastructure and support systems to ensure proper airborne precautions to prevent Mycobacterium tuberculosis transmission, and contact precautions for bacterial infections other than $T B$, focusing on identification and isolation of patients, personal protective equipment and above all excellent hand hygiene practice, lie at the heart of the IPC strategy. Involving our patients by empowering them to remind health professionals to comply with hand hygiene and protective equipment is another valuable piece in the jigsaw. ${ }^{[17]}$

AMR is no longer someone else's problem. It's personal, it's here to stay, and if we don't do something about it now, our patients' morbidity and mortality will increase substantially. It would be good not to hear anyone say 'Nice resolution, shame about the action.'

\section{Marc Mendelson}

Division of Infectious Diseases and HIV Medicine, Department of Medicine, Groote Schuur Hospital and Faculty of Health Sciences, University of Cape Town, Cape Town, South Africa

\section{Malebona Precious Matsoso}

National Department of Health, Pretoria, South Africa

Corresponding author: M Mendelson (marc.mendelson@uct.ac.za)

1. World Health Assembly Resolution WHO 134.R13. Combating antimicrobial resistance including antibiotic resistance. http://apps.who.int/gb/ebwha/pdf_files/EB134/B134_R13-en.pdf (accessed 2 June 2014).

2. World Health Assembly Resolution WHA51.17. Emerging and other communicable diseases: antimicrobial . World Health Assembly Resolution WHA51.17. Emerging and other communicable diseases: anting
resistance. http://apps.who.int/medicinedocs/documents/s16334e/s16334e.pdf (accessed 2 June 2014)

3. World Health Assembly Resolution WHA58.27. Improving the containment of antimicrobial resistance. http://www.searo.who.int/entity/medicines/topics/wha_58_27.pdf (accessed 2 June 2014).

4. Kumarasamy KK, Toleman MA, Walsh TR, et al. Emergence of a new antibiotic resistance mechanism in India, Pakistan, and the UK: A molecular, biological, and epidemiological study. Lancet Infect Dis 2010;10(9):597602. [http://dx.doi.org/10.1016/S1473-3099(10)70143-2]

5. Nordmann P, Cuzon G, Naas T. The real threat of Klebsiella pneumoniae carbapenemase-producing bacteria. Lancet Infect Dis 2003;9(4):228-236. [http://dx.doi.org/10.1016/S1473-3099(09)70054-4]

6. Centers for Disease Control and Prevention, US Department of Health and Human Services. Antibiotic Resistance Threats in the United States, 2013. Atlanta, Ga: CDC, 2013. http://www.cdc.gov/drugresistance/ threat-report-2013/ (accessed 2 June 2014)

7. European Commission. Action plan against the rising threats from antimicrobial resistance. Brussels, 2011. http://ec.europa.eu/dgs/health_consumer/docs/communication_amr_2011_748_en.pdf (accessed 2 June 2014).

8. Cai Y, Chai D, Wang R, Liang B, Bai N. Colistin resistance of Acinetobacter baumannii: Clinical reports, mechanisms and antimicrobial strategies. J Antimicrob Chemother 2012;67(7):1607-1615. [http://dx.doi. org/10.1093/jac/dks084]

9. Brink AJ, Coetzee J, Corcoran C, et al. Emergence of OXA-48 and OXA-181 carbapenemases among Enterobacteriaceae in South Africa and evidence of in vivo selection of colistin resistance as a consequence org/10.1128/JCM.02234-12

10. Klopper M, Warren RM, Hayes C, et al. Emergence and spread of extensively and totally drug-resistant (1903.120246]

11. World Health Organization A

12. World Health Organization. WHO Drug Resistance Report 2012. Geneva: WHO, 2012.

Dondorp AM, Yeung S, White L, et al. Artemisinin resistance: Current status and scenarios for containment. Nat Rev Microbiol 2010;8(4):272-280. [http://dx.doi.org/10.1038/nrmicro2331]

14. Duse A. The Global Antibiotic Resistance Partnership. S Afr Med J 2011;101(8):551.
15. Mendelson M, Whitelaw A, Nicol M, Brink A. Wake up South Africa! The antibiotic horse' has bolted. S Afr Medelson $M$, Whitelaw A,

16. Boyles TH, Whitelaw A, Bamford C, et al. Antibiotic stewardship ward rounds and a dedicated prescription Boyles $\mathrm{TH}$, Whitelaw A, Bamford C, et al. Antibiotic stewardship ward rounds and a dedicated prescription
chart reduce antibiotic consumption and pharmacy costs without affecting inpatient mortality or re-admission rates. PLoS One 2013;8(12):e79747. [http://dx.doi.org/10.1371/journal.pone.0079747]

17. Mendelson M. Patient empowerment as a driver of hand hygiene practice: Time for patients in South Africa to have their say. Southern African Journal of Infectious Diseases 2014;29(1):3-4,

S Afr Med J 2014;104(7):478-479. DOI:10.7196/SAMJ.8534 